\title{
Report on the External Quality Assessment Scheme for Blood Gas (Central Laboratory and Point-of- Care Testing) and Glucose (Point-of-Care Testing) Analysis in Korea (2016-2017)
}

Jinsook Lim ${ }^{1}$, Hee-Jung Chung ${ }^{2}$, Byung Ryul $\mathrm{Jeon}^{3}$, and Gye Cheol Kwon $^{1}$

${ }^{1}$ Department of Laboratory Medicine, Chungnam

National University

Hospital, Daejeon;

${ }^{2}$ Department of Laboratory

Medicine, Kunkuk

University Hospital, Seoul;

${ }^{3}$ Department of Laboratory

Medicine and Genetics,

Soonchunhyang University

Bucheon Hospital, Bucheon,

Korea

\author{
Corresponding author: \\ Gye Cheol Kwon \\ Department of Laboratory \\ Medicine, Chungnam National \\ University Hospital, 282 \\ Munhwa-ro, Jung-gu, Daejeon \\ 35015, Korea \\ Tel: +82-42-280-7799 \\ Fax: +82-42-257-5365 \\ E-mail: kckwon@cnu.ac.kr
}

pISSN: 2384-2458

eISSN: 2288-7261
In the 2016 and 2017 programs for blood gas analysis (BGA) in central laboratory and by point-of-care testing (POCT), and glucose analysis by POCT, external quality assessment of 9,3 , and 1 analytes, respectively, was performed each year. The materials used were commercially available quality control materials, and three levels were used per trial. Based on the information and results from each participating laboratory, statistical analysis was carried out. Results were provided to each laboratory through individual and comprehensive reports. The mean response rates were $96.6 \%, 96.5 \%$, and $95.6 \%$ for BGA in central laboratory, BGA (POCT), and glucose (POCT), respectively. The number of participating laboratories in BGA (central laboratory and POCT) in 2017 was not significantly different from that in 2016. However, in the glucose (POCT) program, the number of registered instruments sharply increased in 2017 as the allowable number of registered instruments was increased from 5 to 30 . The coefficient of variation (CV) did not show any significant differences in $\mathrm{pH}$, sodium, chloride, and ionized calcium of BGA. However, the differences of $\mathrm{CV}$ were found to be relative large between instruments in other analytes of BGA and glucose POCT.

(J Lab Med Qual Assur 2018;40:171-177)

Key Words: Proficiency testing, Blood gas analysis, Point-of-care testing

\section{서론}

1976년 8월 21일 창립된 대한임상검사정도관리협회는 1977 년도에 39 개 병원의 검사실을 대상으로 검사실 간 신빙도조 사를 국내에서 처음으로 실시하였다. 그 중 임상화학분과는 1980년도에 발족되어 일반화학검사 및 혈액가스검사에 대한
신빙도조사사업을 담당하였으며[1-3], 2008년 당화혈색소, 2011년 정확도 기반 지질 및 크레아티닌 프로그램을 추가하 였다[4]. 2016년을 기점으로 분과 중심의 운영체계에서 단위 별 운영체계로 전환이 되었으며, 혈액가스검사 분야가 일반화 학검사 프로그램에서 혈액가스검사 프로그램으로 따로 분리 되는 큰 변화가 있었다[5]. 또한 검사실 이외의 장소에서 이용 


\title{
Journal of LABORATORY MEDICINE and QUALITY ASSURANCE
}

\author{
Jinsook Lim et al • Report of K-EQAS for BGA and Glucose POCT
}

되고 있는 혈액가스검사장비 및 간이혈당검사기기에 대한 외 부정도관리의 요구도가 높아짐에 따라, 검사실 밖에서 운영되 는 혈액가스장비에 대한 평가를 위해 혈액가스 현장검사 그리 고 glucometer에 대한 glucose 현장검사 프로그램이 각각 추 가되었다. 저자들은 2016년부터 2017년까지 실시된 혈액가스 검사, 혈액가스 현장검사 그리고 glucose 현장검사 프로그램의 신빙도조사사업 결과를 분석하여 보고하고자 한다.

\section{재료 및 방법}

\section{1. 대상}

2016년부터 2017년까지 혈액가스검사, 혈액가스 현장검사 그리고 glucose 현장검사 모두 연간 2회에 걸쳐 회차당 3개의 검체에 대해 신빙도조사를 시행하였다. 정도관리물질은 Table 1 의 일정대로 발송되었다. 다수의 검사장비가 운영되는 현장 검사의 특성을 고려하여, 동일 기관에서 여러 장비가 신빙도조 사에 참여할 수 있도록 프로그램을 점진적으로 확장하였다. 혈 액가스 현장검사의 경우 2017년부터 동일 기관에서 6대의 장
비까지 참여가 가능하였다. Glucose 현장검사의 경우 2016년 부터 동일 기관에서 최대 5대까지, 2017년부터는 최대 30대까 지 참여가 가능하였다.

\section{2. 정도관리물질과 검사종목}

혈액가스검사에 대한 정도관리물질은 Liquichek Blood Gas Plus EGL Quality Controls (Bio-Rad Laboratories, Hercules, CA, USA), 혈액가스 현장검사의 경우 Liquichek Blood Gas Plus E Quality Controls (Bio-Rad Laboratories), 그리고 glucose 현장검사의 경우 Liquichek Meter Trax Control (Bio-Rad Laboratories)을 각각 사용하였다(Table 2). 2016년의 경우 1차 및 2 차에 사용된 정도관리물질의 로트번호 가 동일하였으나, 2017년도에는 회차별로 다른 로트번호의 물 질을 사용하였다. 각 프로그램별로 검체는 3가지 농도의 물질 로 구성되었다. 혈액가스의 검사항목은 $\mathrm{pH}$, partial pressure of carbon dioxide $\left(\mathrm{pCO}_{2}\right)$, partial pressure of oxygen $\left(\mathrm{pO}_{2}\right)$, sodium, potassium, chloride, ionized calcium, ionized magnesium, lactic acid의 9종이며, 혈액가스 현장검사의 검

Table 1. Sample shipment schedules for external quality assessments of blood gas analysis (central laboratory and POCT) and glucose (POCT) in the 2016 and 2017 trials

\begin{tabular}{lccc}
\hline \multicolumn{1}{c}{ Trial } & Blood gas analysis (central laboratory) & Blood gas analysis (POCT) & Glucose (POCT) \\
\hline 1st, 2016 & 2016.4 .26$. & 2016.4 .27$. & 2016.5 .31$. \\
2nd, 2016 & 2016.9 .19$. & 2016.9 .19$. & 2016.9 .19$. \\
1st, 2017 & 2017.3 .13$. & 2017.3 .13$. & 2017.3 .13$. \\
2nd, 2017 & 2017.8 .28$. & 2017.8 .28$. & 2017.9 .18$. \\
\hline
\end{tabular}

Abbreviation: POCT, point-of-care testing.

Table 2. Materials used, test items, and sample numbers of external quality assessment schemes for blood gas analysis (central laboratory and POCT) and glucose (POCT)

\begin{tabular}{|c|c|c|c|c|c|}
\hline \multirow{2}{*}{ Program } & \multirow{2}{*}{ Materials used (analytes) } & \multicolumn{4}{|c|}{ No. of sample } \\
\hline & & 1st, 2016 & 2nd, 2016 & 1st, 2017 & 2nd, 2017 \\
\hline \multirow{3}{*}{$\begin{array}{l}\text { Blood gas analysis } \\
\text { (central laboratory) }\end{array}$} & \multirow{3}{*}{$\begin{array}{l}\text { Liquicheck Blood Gas Plus EGL } \\
\text { Quality Controls ( } \mathrm{pH}, \mathrm{pCO}_{2}, \mathrm{pO}_{2} \text {, } \\
\text { sodium, potassium, chloride, } \mathrm{iCa}, \\
\text { iMg, lactic acid) }\end{array}$} & CA-16-01 & CA-16-04 & CA-17-01 & CA-17-04 \\
\hline & & CA-16-02 & CA-16-05 & CA-17-02 & CA-17-05 \\
\hline & & CA-16-03 & CA-16-06 & CA-17-03 & CA-17-06 \\
\hline \multirow{3}{*}{$\begin{array}{l}\text { Blood gas analysis } \\
\text { (POCT) }\end{array}$} & \multirow{3}{*}{$\begin{array}{l}\text { Liquicheck Blood Gas Plus E Quality } \\
\text { Controls }\left(\mathrm{pH}, \mathrm{pCO}_{2}, \mathrm{pO}_{2}\right)\end{array}$} & CAP-16-01 & CAP-16-04 & CAP-17-01 & CAP-17-04 \\
\hline & & CAP-16-02 & CAP-16-05 & CAP-17-02 & CAP-17-05 \\
\hline & & CAP-16-03 & CAP-16-06 & CAP-17-03 & CAP-17-06 \\
\hline \multirow[t]{3}{*}{ Glucose (POCT) } & \multirow{3}{*}{$\begin{array}{l}\text { Liquichek Meter Trax Control } \\
\text { (glucose) }\end{array}$} & GGP-16-01 & GGP-16-04 & CGP-17-01 & CGP-17-04 \\
\hline & & GGP-16-02 & GGP-16-05 & CGP-17-02 & CGP-17-05 \\
\hline & & GGP-16-03 & GGP-16-06 & CGP-17-03 & CGP-17-06 \\
\hline
\end{tabular}

Abbreviations: POCT, point-of-care testing; $\mathrm{pCO}_{2}$, partial pressure of carbon dioxide; $\mathrm{pO}_{2}$, partial pressure of oxygen; iCa, ionized calcium; iMg, ionized magnesium. 


\section{Journal of LABORATORY MEDICINE and QUALITY ASSURANCE}

\section{Jinsook Lim et al • Report of K-EQAS for BGA and Glucose POCT}

사종목은 $\mathrm{pH}, \mathrm{pCO}_{2}, \mathrm{pO}_{2}$ 의 3 종, 그리고 glucose 현장검사는 glucose 단일 종목이었다.

\section{3. 결과 판정 및 분석}

각 기관에서 입력한 결과를 바탕으로 제조사 및 검사장비에 따른 통계분석을 시행하였고, 전체에 해당하는 통계, 동일 제 조사에 따른 기준분류 및 동일 장비를 사용하는 세분류에 따 른 통계를 각각 제시하였다. 각 참여기관에는 개별기관의 평가 자료를 나타내는 기관별 보고서 및 전체 참여기관의 평가결과 를 나타낸 공통보고서를 제공하였다. 기관별 보고서에는 각 기 관의 결과에 대한 전체, 동일한 제조사에 기반한 기준분류 그
리고 동일한 장비에 기반한 세분류에 따른 참여기관 수, 평균 (mean), 표준편차(standard deviation), 변이계수(coefficient of variation), 중앙값(median), 최소값, 최대값 그리고 표준 편차지수(standard deviation index, SDI)를 기록하였다. 그 리고 공통보고서에는 참여한 전체기관, 기준분류 및 세분류 에 대한 참여기관의 수, 평균, 표준편차, 변이계수, 중앙값, 최 소값, 최대값을 제시하였다. 평균, 표준편차 그리고 변이계 수의 경우 해당 분류별 분석에서 75 percentile 값(Q3)과 25 percentile 값(Q1)의 차(Q3-Q1; interquartile range, IQR)의 1.5 배를 초과하여 $\mathrm{Q} 1$ 보다 낮거나 $\mathrm{Q} 3$ 보다 높은 결과값 $(<\mathrm{Q} 1-$ $1.5 \times \mathrm{IQR}$ or $>\mathrm{Q} 3+1.5 \times \mathrm{IQR})$ 을 이상치로 제거한 후 다시

Table 3. Number of invited and participating laboratories for blood gas analysis (central laboratory and POCT) and glucose (POCT)

\begin{tabular}{|c|c|c|c|c|c|c|}
\hline \multirow[b]{2}{*}{ Trial } & \multicolumn{2}{|c|}{$\begin{array}{c}\text { Blood gas analysis (central } \\
\text { laboratory) }\end{array}$} & \multicolumn{2}{|c|}{ Blood gas analysis (POCT) } & \multicolumn{2}{|c|}{ Glucose (POCT) } \\
\hline & $\begin{array}{c}\text { No. of } \\
\text { laboratories } \\
\text { invited }\end{array}$ & $\begin{array}{c}\text { Participating } \\
\text { laboratories (\%) }\end{array}$ & $\begin{array}{c}\text { No. of } \\
\text { laboratories } \\
\text { invited }\end{array}$ & $\begin{array}{c}\text { Participating } \\
\text { laboratories (\%) }\end{array}$ & $\begin{array}{c}\text { No. of } \\
\text { laboratories } \\
\text { invited }\end{array}$ & $\begin{array}{c}\text { Participating } \\
\text { laboratories (\%) }\end{array}$ \\
\hline 1st, 2016 & 367 & $358(97.5)$ & 154 & $150(97.4)$ & 214 & $196(91.6)$ \\
\hline 2nd, 2016 & 367 & $355(96.7)$ & 154 & $148(96.1)$ & 214 & $207(96.7)$ \\
\hline 1st, 2017 & 390 & $370(94.9)$ & 160 & $149(93.1)$ & 249 & $239(96.0)$ \\
\hline 2nd, 2017 & 390 & $380(97.4)$ & 160 & $159(99.4)$ & 249 & $244(98.0)$ \\
\hline
\end{tabular}

Abbreviation: POCT, point-of-care testing.

Table 4. Number of participating laboratories for blood gas analysis (central laboratory) and instruments for blood gas analysis (POCT) and glucose (POCT) in 2016 and 2017

\begin{tabular}{|c|c|c|c|c|c|}
\hline \multirow{2}{*}{ Program } & \multirow{2}{*}{ Analyte } & \multicolumn{4}{|c|}{ Trial } \\
\hline & & 1st, 2016 & 2nd, 2016 & 1st, 2017 & 2nd, 2017 \\
\hline \multirow[t]{9}{*}{ Blood gas analysis } & $\mathrm{pH}$ & 358 & 355 & 370 & 380 \\
\hline & $\mathrm{pCO}_{2}$ & 358 & 355 & 370 & 380 \\
\hline & $\mathrm{pO}_{2}$ & 358 & 355 & 370 & 380 \\
\hline & Sodium & 183 & 175 & 187 & 196 \\
\hline & Potassium & 183 & 175 & 187 & 196 \\
\hline & Chloride & 138 & 130 & 137 & 141 \\
\hline & $\mathrm{iCa}$ & 203 & 207 & 227 & 239 \\
\hline & $\mathrm{iMg}$ & & 44 & 47 & 48 \\
\hline & Lactic acid & & 56 & 81 & 90 \\
\hline \multirow[t]{3}{*}{ Blood gas analysis (POCT) } & $\mathrm{pH}$ & 150 & 148 & 197 & 207 \\
\hline & $\mathrm{pCO}_{2}$ & 150 & 148 & 197 & 207 \\
\hline & $\mathrm{pO}_{2}$ & 150 & 148 & 197 & 207 \\
\hline Glucose (POCT) & Glucose & 755 & 808 & 2,216 & 2,312 \\
\hline
\end{tabular}

The bold type of the table shows that a program has been established to allow multiple instruments to participate in a single institution. Abbreviations: POCT, point-of-care testing; $\mathrm{pCO}_{2}$, partial pressure of carbon dioxide; $\mathrm{pO}_{2}$, partial pressure of oxygen; iCa, ionized calcium; iMg, ionized magnesium. 
Jinsook Lim et al • Report of K-EQAS for BGA and Glucose POCT

분석한 값을 제시하였다. 세분류를 기준으로 해당 분류에 속하 는 기관 수가 10 개 미만인 경우에는 평균, 표준편차, 변이계수, $\mathrm{SDI}$ 를 제시하지 않았으며, 기관 수가 3개 미만인 경우에는 중 앙값도 제시하지 않았다. 또한 세분류를 기준으로 해당 분류에 속하는 기관 수가 1 개인 경우에는 공통보고서에 포함되지 않
았다. 판정은 기준분류 혹은 세분류에서 $\mathrm{SDI}$ 가 3 미만인 경우 적합(acceptable)하다고 하였다.

Table 5. List of manufacturers and instruments used for blood gas analysis (central laboratory)

\begin{tabular}{|c|c|c|c|c|c|}
\hline \multirow{2}{*}{ Manufacturer } & \multirow{2}{*}{ Instrument } & \multicolumn{4}{|c|}{ Trial } \\
\hline & & 1st, 2016 & 2nd, 2016 & 1st, 2017 & 2nd, 2017 \\
\hline Edan Instruments Inc. & i15 & & & & 2 \\
\hline \multirow[t]{2}{*}{ Instrumentation Laboratory } & GEM Premier 3000 & 13 & 10 & 10 & 8 \\
\hline & GEM Premier 3500 & 34 & 36 & 43 & 47 \\
\hline i-SENS & i-Smart 300 & 2 & 5 & 5 & 7 \\
\hline MEDICA Corp. & EasyBloodGas & 2 & 2 & 3 & 2 \\
\hline \multirow[t]{7}{*}{ Nova Biomedical Corp. } & Stat Profile CCX 1, 2, 3, 4, 1+, 2+, 3+, 4+ & 26 & 22 & 22 & 18 \\
\hline & Stat Profile $\mathrm{pHOx}$ & 13 & 12 & 11 & 7 \\
\hline & Stat Profile pHOx Basic & 3 & 1 & 2 & 1 \\
\hline & Stat Profile pHOx Ultra with CO-Oximeter & 20 & 19 & 21 & 24 \\
\hline & Stat Profile pHOx Ultra without CO-Oximeter & 18 & 20 & 24 & 25 \\
\hline & Stat Profile Prime ABG & & & & 1 \\
\hline & Stat Profile Prime CCS & & 1 & 1 & 4 \\
\hline \multirow[t]{4}{*}{ OPTI Medical Systems Inc. } & OPTI CCA & 3 & 3 & 2 & 6 \\
\hline & OPTI CCA-TS & 2 & 2 & 2 & 2 \\
\hline & OPTI CCA-TS2 & & & 1 & 2 \\
\hline & Others & 1 & 1 & & \\
\hline Radiometer & ABL5 & 4 & 3 & 2 & 2 \\
\hline Roche & Others & & & 1 & \\
\hline Siemens Healthcare & RAPIDLab 248/348 & 20 & 21 & 19 & 18 \\
\hline \multirow[t]{4}{*}{ Diagnostics Inc. } & RAPIDLab 348EX & 9 & 10 & 13 & 13 \\
\hline & RAPIDLab 1245/1260/1265 & 45 & 45 & 44 & 43 \\
\hline & RAPIDPoint 400/405 & 6 & 5 & 6 & 5 \\
\hline & RAPIDPoint 500 & 9 & 10 & 11 & 16 \\
\hline Others & Others & 1 & 1 & 2 & 1 \\
\hline Total & & 358 & 355 & 370 & 380 \\
\hline
\end{tabular}




\section{결과 및 고찰}

2016년에서부터 2017년까지 혈액가스검사, 혈액가스 현장 검사 그리고 glucose 현장검사에 대한 회신기관 수 및 회신율 은 Table 3에 각각 표시하였으며, 각 검사항목별 참여기관 혹
은 참여장비의 수는 Table 4에 나타내었다. Table 4에서 현장 검사 프로그램들의 경우 참여기관 수가 매년 급격하게 증가 하는 것처럼 보이는데, 이것은 동일 기관에서 여러 장비를 신 빙도조사에 참여할 수 있도록 프로그램을 확장하였기 때문이 다. 참여기관 수의 증가를 2016년과 2017년 마지막 회차 회신

Table 6. List of manufacturers and instruments participated in external quality assessment program for blood gas analysis (point-of-care testing)

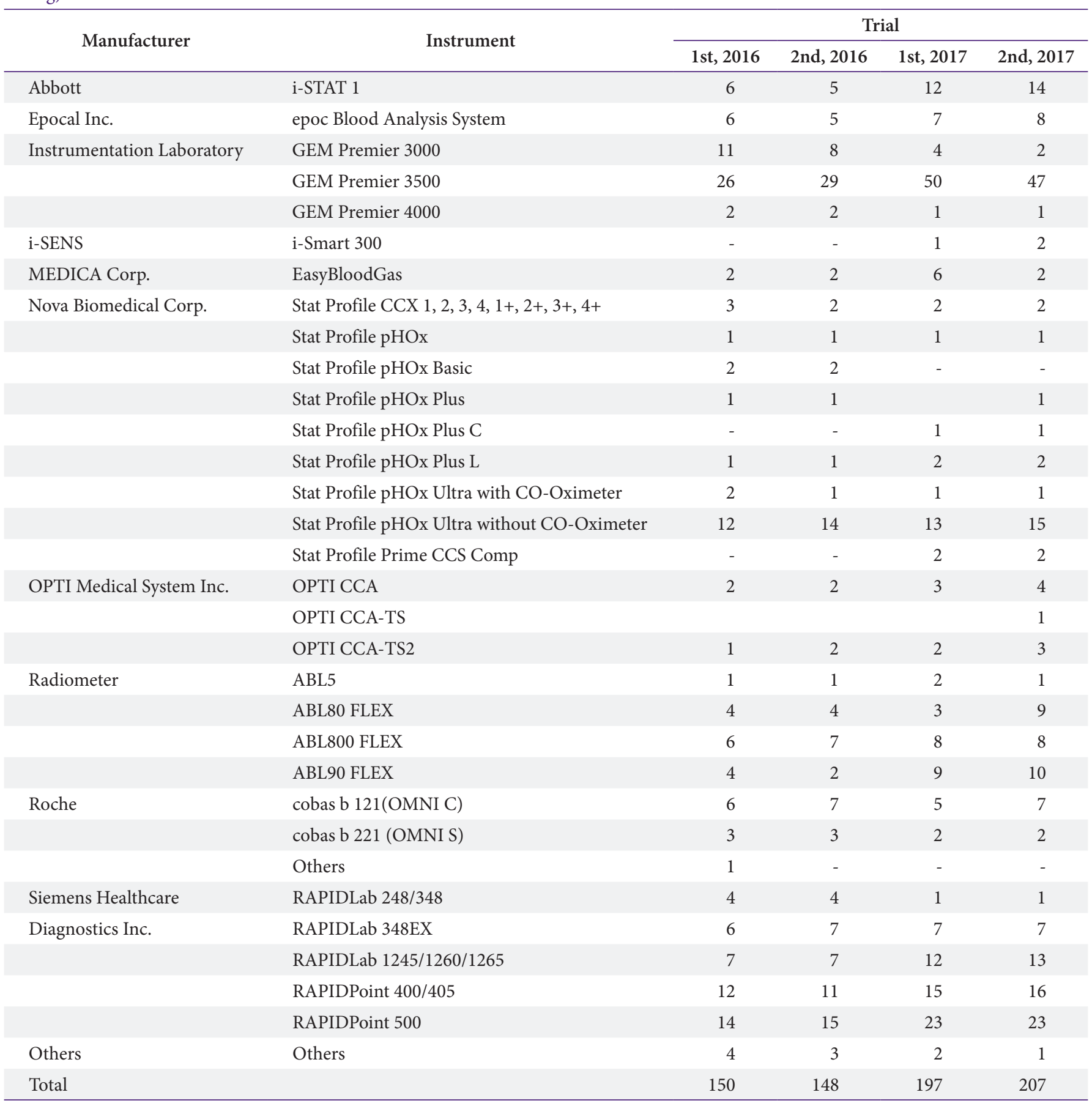


기관 수를 기준으로 계산하였을 때, 2017년도 혈액가스검사 프로그램의 경우 25 기관이 증가하여 $7.0 \%$ (25/355기관), 혈 액가스 현장검사 프로그램의 경우 11 기관이 증가하여 $7.4 \%$ (11/148기관), glucose 현장검사 프로그램의 경우 37기관
이 증가하여 $17.9 \%$ (37/207기관)의 증가추세를 나타내었다. Glucose 현장검사 프로그램에서 참여기관의 수가 가장 큰 폭 으로 증가하였다.

혈액가스검사, 혈액가스 현장검사 그리고 glucose 현장검사

Table 7. List of manufacturers and instruments participated in external quality assessment program for glucose (point-of-care testing)

\begin{tabular}{|c|c|c|c|c|c|}
\hline \multirow{2}{*}{ Manufacturer } & \multirow{2}{*}{ Instrument } & \multicolumn{4}{|c|}{ Trial } \\
\hline & & 1st, 2016 & 2nd, 2016 & 1st, 2017 & 2nd, 2017 \\
\hline \multirow[t]{2}{*}{ Allmedicus } & GlucoDr. Plus (AGM-3000/B) & 19 & 26 & 78 & 66 \\
\hline & GlucoDr. Auto (AGM-4000) & & & & 1 \\
\hline \multirow[t]{2}{*}{ Arkray Inc. } & Glucocard 01 & 5 & 5 & 14 & 14 \\
\hline & Glucocard II & & & 2 & 2 \\
\hline \multirow[t]{2}{*}{ Ascensia } & Breeze 2 & 33 & 36 & 101 & 51 \\
\hline & Contour plus & 2 & 9 & 20 & 18 \\
\hline \multirow[t]{4}{*}{ Infopia } & GlucoLab & & & 1 & 1 \\
\hline & Glucolab Auto Coding & & & & 7 \\
\hline & Gluneo Lite & 25 & 25 & 2 & 2 \\
\hline & Others & & & 58 & 58 \\
\hline \multirow[t]{10}{*}{ i-SENS } & BAROZen & 27 & 31 & 45 & 53 \\
\hline & BAROZen H plus & 24 & 54 & 97 & 125 \\
\hline & BAROZen $\mathrm{H}$ & 104 & 93 & 138 & 139 \\
\hline & BAROzen $\mathrm{H}$ expert plus & & & 17 & 30 \\
\hline & BAROzen $\mathrm{H}$ expert & & & 179 & 260 \\
\hline & CareSens II & 8 & 7 & 7 & 7 \\
\hline & CareSens N & 16 & 17 & 57 & 61 \\
\hline & CareSens N Voice & 9 & 10 & 58 & 59 \\
\hline & CareSens PRO & & & 26 & 46 \\
\hline & Others & 7 & 12 & 13 & 8 \\
\hline \multirow[t]{2}{*}{ Johnson \& Johnson } & ONETOUCH Verio & 5 & & & \\
\hline & ONETOUCH VerioPro+ & 15 & 12 & 17 & 17 \\
\hline \multirow[t]{4}{*}{ Roche } & Accu-Chek Active & 37 & 60 & 129 & 130 \\
\hline & Accu-Chek Inform II & 176 & 157 & 400 & 358 \\
\hline & Accu-Chek Performa & 91 & 130 & 460 & 508 \\
\hline & Others & 24 & 4 & 2 & 2 \\
\hline \multirow[t]{6}{*}{ SD Biosensor } & SD CHECK GOLD (Meter 01GM10) & 5 & 5 & & \\
\hline & SD CodeFree (Meter 01GM11) & 35 & 35 & 62 & 56 \\
\hline & SD CodeFree (System 01GC112) & & & 9 & 9 \\
\hline & SD GlucoNavii Link 0.3 (Meter 01GM12) & & & 30 & 33 \\
\hline & SD GlucoNavii NFC (Meter 01GM40) & 15 & 15 & 78 & 87 \\
\hline & Others & 1 & 3 & 8 & 7 \\
\hline Wise Meditech & Wisecheck & 3 & 3 & 3 & 1 \\
\hline Others & Others & 69 & 59 & 105 & 96 \\
\hline Total & & 755 & 808 & 2,216 & 2,312 \\
\hline
\end{tabular}


의 측정장비의 분포는 Tables 5-7에, 그리고 각 프로그램별 검 사항목별 평균, 표준편차 그리고 변이계수는 Supplemental Tables 1-13에 나타나 있다. $\mathrm{pH}$ 의 경우 대부분의 제조사의 장 비군에서 변이계수가 매우 낮은 것으로 나타났으며, 검사장비 별로 검사실 간 정밀도에 큰 차이가 없어, 장비들의 성능이 상 향평준화된 것으로 판단된다. 현재 사용하고 있는 statistical limit을 적용할 경우 정밀도가 좋은 경우 임상적으로 의미 없 는 차이까지 문제가 있는 것처럼 보이게 할 수 있으므로 실 제 기관의 수행능(performance)을 평가하는 데 제약이 따를 수 있다[5]. 따라서 조금 더 느슨한 기준으로 재조정이 필요 할 것으로 판단된다. $\mathrm{pCO}_{2}$ 와 $\mathrm{pO}_{2}$ 의 경우 $\mathrm{pH}$ 만큼 정밀도가 좋 지 않았고, 장비 간 차이가 있는 것으로 나타났으며, 검사실에 서 측정결과와 현장검사로 측정한 결과 모두 비슷하게 나타났 다. 검사실 혈액가스검사장비로 측정한 sodium, potassium, chloride, ionized calcium의 경우 장비별로 정밀도의 차 이가 크지 않은 것으로 나타났으나, lactic acid 및 ionized magnesium의 경우 정밀도의 차이가 큰 것으로 나타났다.

Glucose 현장검사의 경우 참여장비의 수가 2016년 1차 755 개로 시작하여 2017년 2차에서는 무려 2,312개로 증가하였 다. 전년 대비 참여장비 수 비율을 매년 마지막 회차 회신장 비 수를 기준으로 계산하였을 때, 2017년 혈액가스 현장검사 프로그램의 경우 참여 장비 수는 59 개가 증가하여 전년 대비 $39.9 \%$ (59/148대), glucose 현장검사 프로그램의 경우 1,504 개가 증가하여 전년 대비 $186.1 \%$ (1,504/808대)의 증가를 나 타내었다. Glucose 현장검사 프로그램의 경우 2017년부터 동 일 기관에서 최대 30 대까지 신빙도조사에 참여 및 결과관리가 가능하도록 프로그램을 확장한 것이 급격한 증가의 주된 원인 으로 파악된다. 그러나 간이혈당검사기기가 많은 병원에서 널 리 사용되고 있음에도 불구하고 glucose 현장검사 참여기관 의 수(2017년 2회차 244기관)는 혈액가스검사(2017년 2회차 380 기관)에 비해 적은 것으로 나타났으며, 2016년 임상화학프 로그램 glucose 항목에 대한 참여기관 수가 1,263 임을 고려할 때〔6], 아직 참여하지 않은 기관이 많을 것으로 예상된다. 따 라서 기관들의 더 적극적인 참여가 필요하며 이를 통해 검사의 질을 높일 수 있을 것으로 기대한다. Glucose 현장검사의 경우 검사실 간 정밀도의 차이가 제조사별로 큰 것으로 나타났으며,
같은 제조사 내에서도 장비별로 차이를 나타내었다. Glucose 검사의 경우 중앙검사실 장비의 변이계수와 비교하면 glucose 현장검사 변이계수의 평균이 더 높은 것으로 파악된다. 이처럼 변이계수가 큰 경우 SDI는 상대적으로 작아질 수 있으므로 결 과해석 시 반드시 고려할 필요가 있다[6].

\section{SUPPLEMENTARY MATERIALS}

Supplementary materials can be found via https://doi. org/10.15263/jlmqa.2018.40.4.171.

\section{REFERENCES}

1. Tchai BS, Cho HI, Kim SI, Park MH, Moon YH, Cho WH, et al. Report on quality assessment of clinical chemistry in Korea 1978-1980. J Lab Med Qual Assur 1981;3:315.

2. Min WK, Ko C, Kim KD, Kim YK, Kim JH, Kim JQ, et al. Annual report on external quality assessment in clinical chemistry in Korea (2008). J Lab Med Qual Assur 2009;31:1-12.

3. Min WK, Ko C, Kim KD, Kim YK, Kim JH, Kim JQ, et al. Annual report on external quality assessment in clinical chemistry in Korea (2009). J Lab Med Qual Assur 2010;32:1-10.

4. Song J, Kwon KC, Kim JH, Kim JW, Min WK, Lee SY, et al. Annual report on external quality assessment in metabolic disorders in Korea (2009). J Lab Med Qual Assur 2010;32:131-46.

5. Kristensen GB, Meijer P. Interpretation of EQA results and EQA-based trouble shooting. Biochem Med (Zagreb) 2017;27:49-62.

6. Lee YW, Jeon BR, Kim JG, Jun SH, Yun YM, Chun S, et al. Annual report on the external quality assessment scheme for routine clinical chemistry in Korea (2016). J Lab Med Qual Assur 2017;39:61-75. 